02

\title{
Конформеры и электронные спектры дансиламида: экспериментальные и теоретические исследования
}

\author{
() М.С. Федоров, Н.И. Гиричева, Е.А. Лапыкина, М.С. Кораблева
}

Ивановский государственный университет, 153025 Иваново, Россия

e-mail: fms1989@mail.ru

Поступила в редакцию 18.12.2017 г.

В окончательной редакции 01.03.2018 г.

\begin{abstract}
Рассмотрены особенности геометрического и электронного строения шести возможных конформеров дансиламида $\left.\mathrm{CH}_{3}\right)_{2} \mathrm{~N}-\mathrm{C}_{10} \mathrm{H}_{6}-\mathrm{SO}_{2} \mathrm{NH}_{2}$. Методом TDDFT выполнены расчеты электронных спектров поглощения (ЭСП) конформеров дансиламида в свободном состоянии и с учетом влияния водного растворителя (РСМ). Показано, что в результате учета сольватации в электронных спектрах поглощения конформеров проявляется батохромный эффект, а различие в значениях $\lambda$ конформеров достигает $16 \mathrm{~nm}$. Рассчитанные ЭСП в водной среде качественно соответствуют полученным экспериментальным спектрам. Выполнен анализ молекулярных орбиталей, вовлеченных в первые три электронные перехода конформеров. Предполагается, что уширение полосы с $\lambda_{\max }=326 \mathrm{~nm}$ в экспериментальном спектре может быть обусловлено наличием конформеров дансиламида в водном растворе. Показано, что рассчитанные значения $\lambda$ в ЭСП дансиламида в среде водного растворителя существенно зависят от вида функционала электронной плотности.
\end{abstract}

DOI: $10.21883 /$ OS.2018.07.46263.299-17

\section{Введение}

Производные нафталина интересны с практической точки зрения благодаря своей способности к флуоресценции. Так, 5-(диметиламино)нафталин-1-сульфонамид $\left(\left(\mathrm{CH}_{3}\right)_{2} \mathrm{~N}-\mathrm{C}_{10} \mathrm{H}_{6}-\mathrm{SO}_{2} \mathrm{NH}_{2}\right.$, дансиламид) наряду с дансилхлоридом используется в биохимии и химии для флуоресцентного мечения веществ, например, аминокислот и нуклеотидов. Он является молекулой-зондом, которая проявляет сольватохромизм и высокие квантовые выходы излучения и является основной структурой, присутствующей во многих флуоресцентных биосенсорах, метчиках и супрамолекулярных системах [1-3]. Разработка новых лигандов для распознавания нуклеотидов и нуклеиновых кислот на основе дансиламида и его производных является актуальной задачей, и один из первых шагов к ее решению - детальное исследование свойств и различных характеристик молекулы дансиламида.

Кроме того, ряд производных дансиламида широко исследуется на предмет проявления биологической активности [4]. Например, эти соединения были исследованы в качестве ингибиторов таких ферментов, как тромбин, тирозил-ДНК-фосфодиэстераза, метилтрансфера лизинов гистонов, альдолаза, бета-лактамазы AmpC типа и других $[2,4-7]$.

В настоящей работе выполнено исследование электронных спектров поглощения (ЭСП) дансиламида с помощью экспериментальных (UV-Vis-спектроскопия водного раствора) и теоретических методов (DFT, TDDFT). Причем теоретическое моделирование ЭСП проводилось с учетом конформационного состава дансиламида как в газовой фазе, так и в среде водного растворителя (учет сольватации в рамках модели РСМ). Выполнено сравнение геометрических и электронных характеристик конформеров молекулы в свободном и сольватированном состояниях.

Известно, что электронные спектры дансиламида и соединений с дансильной группой $\left(\left(\mathrm{CH}_{3}\right)_{2} \mathrm{~N}-\mathrm{C}_{10} \mathrm{H}_{6}-\right.$ $\left.-\mathrm{SO}_{2}-\mathrm{X}\right)$ чувствительны к полярности растворителя. Так, в работе [8] показано, что максимум полосы поглощения для дансиламида наблюдается при $332 \mathrm{~nm}$ в случае протонных растворителей (метанол, этанол, пропанол и др.). В случае апротонных растворителей он сдвигается, например, в дихлорметане к $343 \mathrm{~nm}$. Для неполярных растворителей (бензол, гексан, диоксан и др.) максимум полосы поглощения наблюдается при $338 \mathrm{~nm}$. В электронных спектрах флуоресценции влияние растворителя проявляется сильнее. Основная тенденция спектров флуоресценции - увеличение батохромного сдвига с увеличением полярности растворителя. Максимум эмиссии наблюдался при $515 \mathrm{~nm}$ в метаноле и $478 \mathrm{~nm}$ в бензоле.

Факторы, определяющие направление и величину сдвига максимумов полос поглощения в ЭСП при взаимодействии соединений с тем или иным растворителем, могут быть различны. Так, в работе [9] рассмотрено влияние релаксации геометрических параметров молекул на сольватохромные сдвиги в электронных спектрах органических соединений. Однако для нежестких молекул, имеющих несколько низкоэнергетических конформеров, кроме вопроса о геометрической релаксации возникает вопрос о проявлении конформационных свойств молекулы в растворителе. 
Молекула дансиламида имеет несколько нежестких торсионных координат, конформационные свойства свободной молекулы дансиламида исследованы в нашей предыдущей работе [10] с помощью теоретических методов DFT(B3LYP)/cc-pVTZ, MP2/cc-pVTZ. Было определено, что молекула имеет 6 конформеров, отличающихся друг от друга взаимной ориентацией групп $-\mathrm{SO}_{2} \mathrm{NH}_{2}$ и $-\mathrm{N}\left(\mathrm{CH}_{3}\right)_{2}$. Геометрическое строение конформеров дансиламида, а также их относительные энергии и дипольные моменты (DFT(B3LYP)/cc-pVTZ) представлены на рис. 1. В газовой фазе преобладает конфомер I, однако при этом сразу четыре конформера (I, III, IV и VI) имеют довольно близкую энергию. В работе также показано, что строение наиболее выгодного конформера для свободной молекулы может значительно отличаться от структур, стабилизированных в кристалле. Каждый из конформеров свободной молекулы с разной вероятностью встречается в разных сокристаллических структурах дансиламида. Причем чем больше межмолекулярное взаимодействие в кристаллах, тем больше изменяется геометрия конформера свободной молекулы. В растворителе возникает ситуация, промежуточная между ситуацией в кристалле (стабилизирована структура, близкая к одному из конформеров) и в газовой фазе (существует равновесная смесь конформеров).

Возникают вопросы: могут ли существовать конформеры дансиламида в среде растворителя, как изменяется их строение, какое влияние это оказывает на ЭСП? Следует отметить, что потенциальные барьеры переходов между конформерами в газовой фазе не превышают $4 \mathrm{kcal} / \mathrm{mol}$ [10]. Кроме того, благодаря межмолекулярному взаимодействию в растворителе, эти конформационные переходы могут значительно облегчиться, что может привести к тому, что в растворителе, так же как в газовой фазе, будет присутствовать смесь конформеров.

\section{Эксперимент}

Регистрация экспериментальных ЭСП проводилась с использованием УВИ-спектрофотометра СФ-56 с термостатируемой ячейкой. Спектральные измерения проводились при температуре $298 \mathrm{~K}$ в диапазоне $190-1100 \mathrm{~nm}$, шаг дискретизации $1 \mathrm{~nm}$, время измерения $0.03 \mathrm{~s}$, ширина щели $3 \mathrm{~nm}$. Предел абсолютной погрешности спектрофотометра при установке длин волн $\pm 1 \mathrm{~nm}$. Измерения проводились для сильно разбавленных водных растворов дансиламида $\left(4 \cdot 10^{-4} \mathrm{~mol} / 1\right.$ и $\left.2 \cdot 10^{-4} \mathrm{~mol} / \mathrm{l}\right)$ с использованием кварцевой кюветы с нормальной толщиной слоя в $1 \mathrm{~cm}$. Коммерческий препарат дансиламид (Aldrich) использовался без дополнительных очисток, его чистота составляла $99 \%$.

\section{Детали квантово-химических расчетов}

При выполнении квантово-химических расчетов (пакет Gaussian-09 [11]) был использован метод DFT (функционалы B3LYP и BMK) с базисными наборами 6-311++ $\mathrm{G}^{* *}$, cc-pVTZ. Для всех стационарных точек на поверхности потенциальной энергии основного электронного состояния исследуемых молекул выполнена полная оптимизация геометрических параметров и расчет частот колебаний. При моделировании ЭСП был использован подход TDDFT. Определялась длина волны поглощения для шести низкоэнергетических переходов. Метод TDDFT - мощный инструмент для изучения возбужденных состояний и моделирования спектров абсорбции и эмиссии органических соединений, который дает хорошо согласующиеся с экспериментальными результаты при относительно небольших затратах компьютерного времени [12-17]. Визуализация геометрических структур выполнена с помощью программы ChemCraft [18].

\section{Результаты и их обсуждение}

\section{Характеристики конформеров молекулы дансиламида}

Шесть конформеров молекулы дансиламида можно разделить на 3 пары, основываясь на особенностях их строения. Конформеры I и III имеют „заслоненную“ конфигурацию группы $-\mathrm{SO}_{2} \mathrm{NH}_{2}$, когда связи $\mathrm{N}-\mathrm{H}$ аминогруппы заслоняют связи $\mathrm{S}=\mathrm{O}$ (рис. 1), условно назовем их „заслоненными“. Конформеры IV и VI имеют „шахматное“ расположение связей $\mathrm{N}-\mathrm{H}$ относительно связей $\mathrm{S}=\mathrm{O}$ группы $-\mathrm{SO}_{2} \mathrm{NH}_{2}-$ условно назовем их „шахматными“. Для обеих групп конформеров характерно такое положение группы $-\mathrm{SO}_{2} \mathrm{NH}_{2}$, когда связь $\mathrm{S}-\mathrm{N}$ располагается почти ортогонально относительно плоскости нафталинового фрагмента. Для конформеров II и $\mathrm{V}$ связь $\mathrm{S}-\mathrm{N}$ лежит практически в плоскости нафталинового фрагмента, условно назовем эти конформеры „плоскими“. Из рис. 1 видно, что „шахматные“ конформеры IV и VI и „заслоненные“ конформеры I и III имеют близкую относительную энергию и являются низкоэнергетическими, „плоские“ конформеры II и V имеют более высокую относительную энергию.

Поскольку в следующей части нашей работы будет рассмотрена длинноволновая область ЭСП дансиламида, то на рис. 2 показаны пять „приграничных“ молекулярных орбиталей (МО) одного из конформеров. Причем, несмотря на существенно отличающееся геометрическое строение конформеров, их соответствующие МО имеют близкие энергии и вид. Так, энергии В3МО для всех конформеров варьируют от -5.88 до $-6.08 \mathrm{eV}$, энергии $\mathrm{HCMO}-$ от -1.80 до $-2.04 \mathrm{eV}$, энергии $\mathrm{HCMO}+1-$ от -0.94 до $-1.12 \mathrm{eV}$, энергии ВЗМО-1 - от -6.95 до -7.14 и энергии ВЗМО-2 - от -7.17 до $-7.37 \mathrm{eV}$. Как 


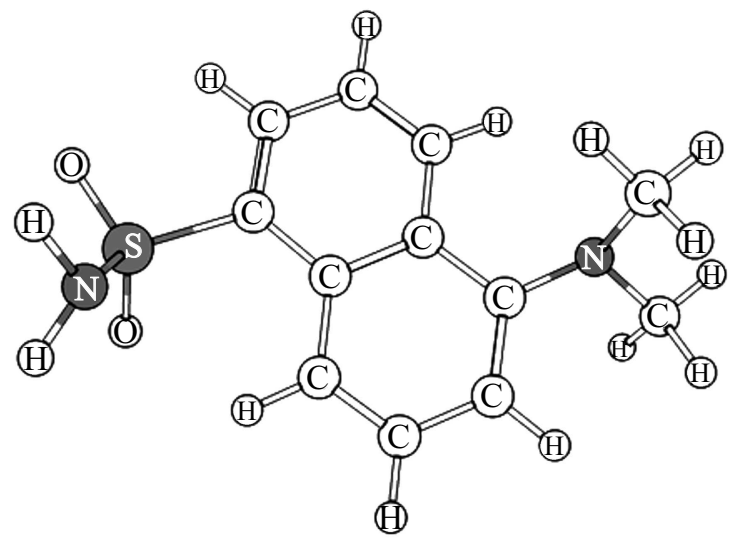

Conformer I

$$
\Delta E_{\text {gas }}=0 ; \Delta E_{\text {water }}=0.72
$$$$
\mu_{\text {gas }}=5.11 ; \mu_{\text {water }}=7.86
$$

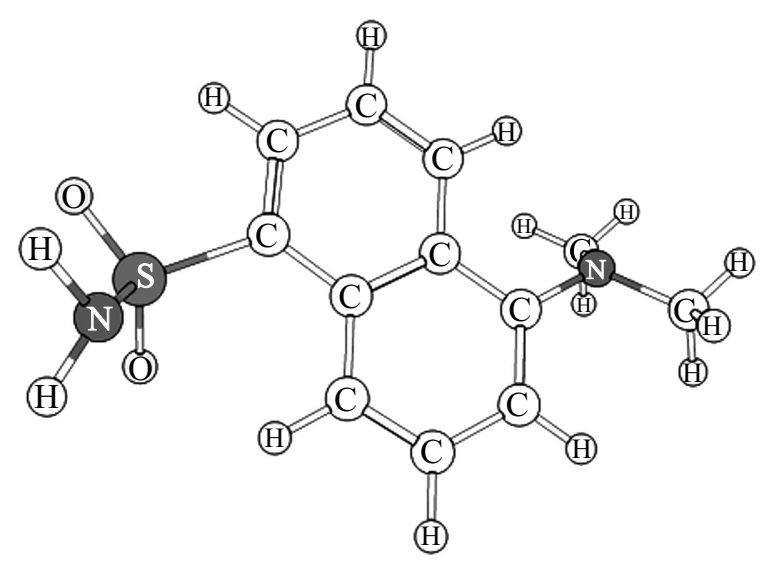

Conformer III

$$
\begin{aligned}
\Delta E_{\text {gas }} & =0.06 ; \Delta E_{\text {water }}=0.75 \\
\mu_{\text {gas }} & =4.65 ; \mu_{\text {water }}=6.84
\end{aligned}
$$

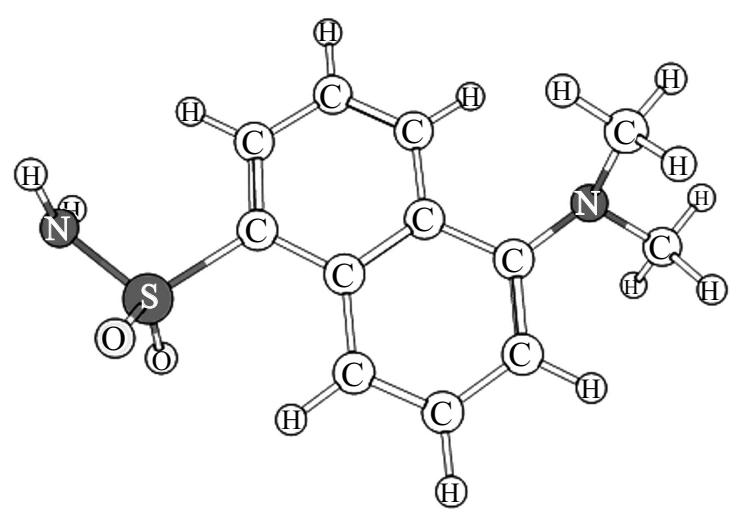

$$
\begin{gathered}
\text { Conformer } \mathrm{V} \\
\Delta E_{\text {gas }}=2.95 ; \Delta E_{\text {water }}=1.25 \\
\mu_{\text {gas }}=5.50 ; \mu_{\text {water }}=8.53
\end{gathered}
$$

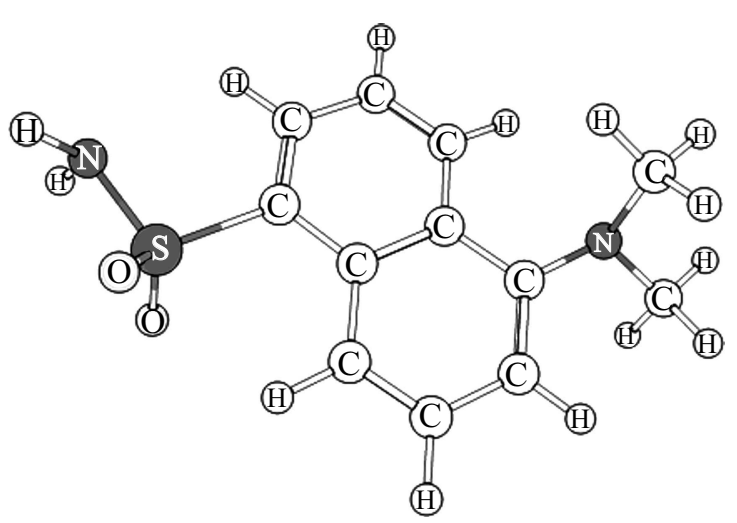

Conformer II

$$
\begin{aligned}
\Delta E_{\text {gas }} & =1.13 ; \Delta E_{\text {water }}=1.73 \\
\mu_{\text {gas }} & =3.67 ; \mu_{\text {water }}=5.86
\end{aligned}
$$

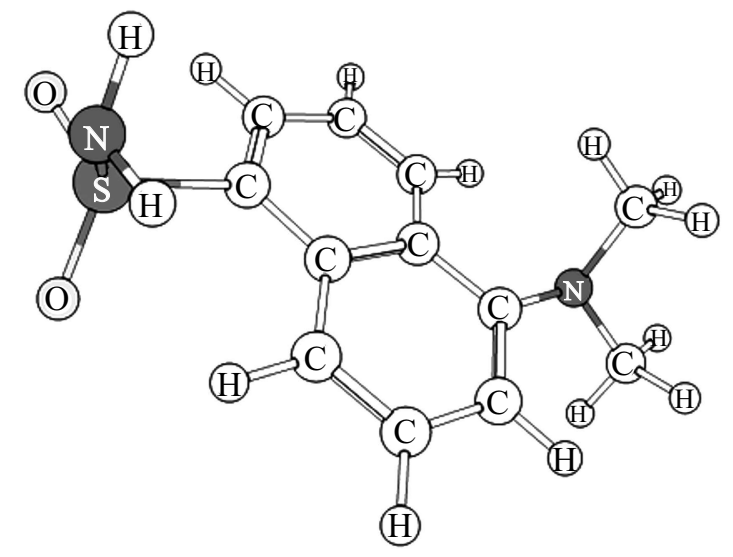

Conformer IV

$$
\begin{aligned}
\Delta E_{\text {gas }}=0.31 ; \Delta E_{\text {water }}=0 \\
\mu_{\text {gas }}=7.20 ; \mu_{\text {water }}=10.44
\end{aligned}
$$

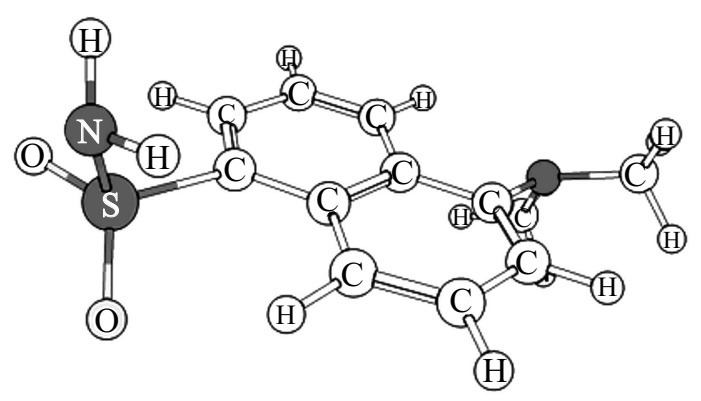

Conformer VI

$$
\begin{aligned}
\Delta E_{\text {gas }} & =0.31 ; \Delta E_{\text {water }}=0.04 \\
\mu_{\text {gas }} & =6.86 ; \mu_{\text {water }}=9.72
\end{aligned}
$$

Рис. 1. Геометрическое строение конформеров свободной молекулы дансиламида с указанием значений относительной энергии $(\Delta E, \mathrm{kcal} / \mathrm{mol})$ и дипольного момента $(\mu, D)[10]$.

видно из рис. 2, ВЗМО-2 является $\pi$-орбиталью нафталинового остова, в ВЗМО-1, так же как и в ВЗМО, существенные вклады вносят атомные орбитали электронодо- норного заместителя $-\mathrm{N}\left(\mathrm{CH}_{3}\right)_{2}$ и $p_{\pi}$-орбитали атомов углерода нафталинового остова. В свою очередь НСМО представляет собой комбинацию из $p_{\pi}$-орбиталей ато- 


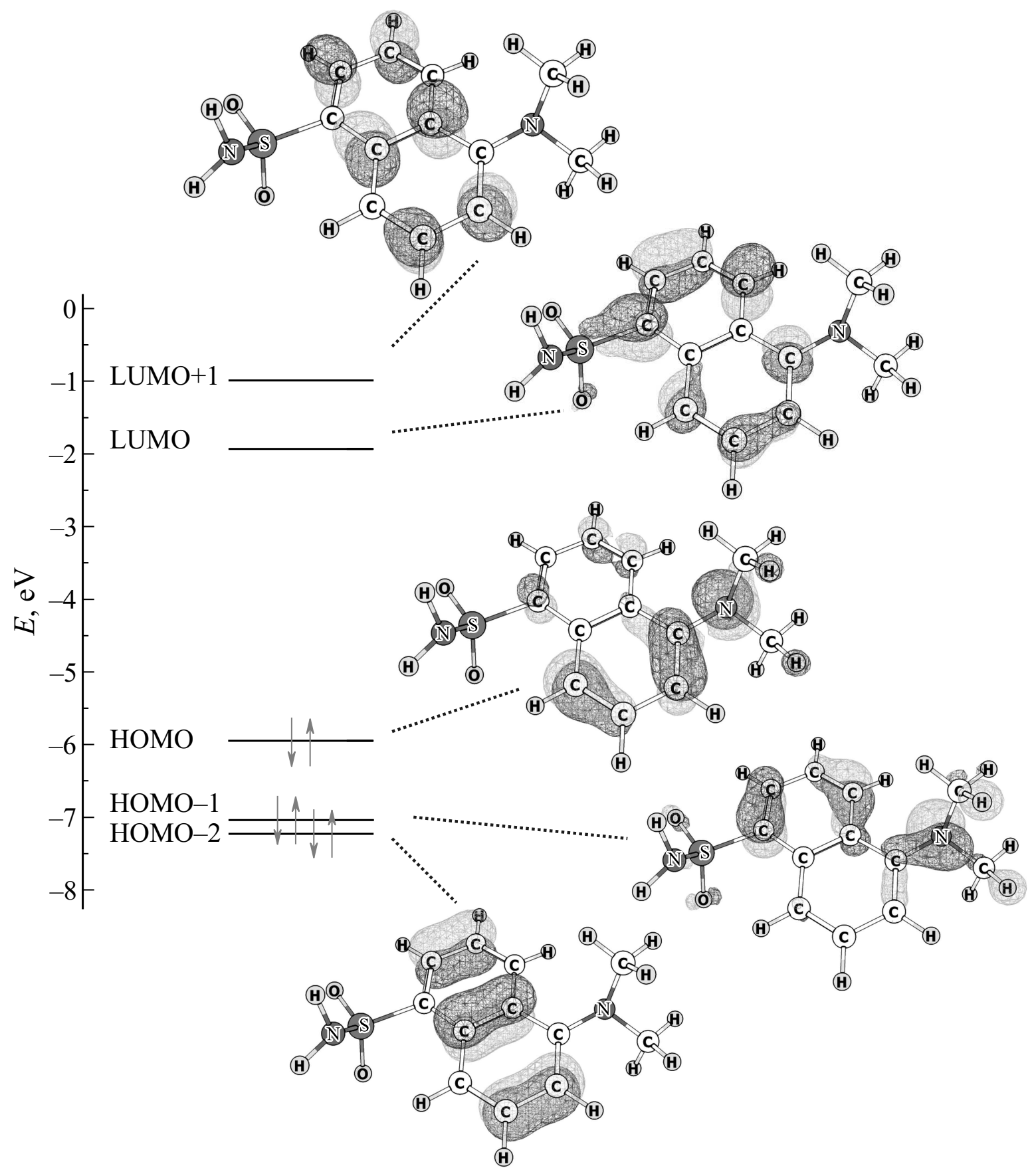

Рис. 2. Энергии и вид граничных и „приграничных“ МO конформера I молекулы дансиламида.

мов углерода нафталинового остова с участием атомных орбиталей серы, относящейся к электроноакцепторному заместителю $-\mathrm{SO}_{2} \mathrm{NH}_{2}, \mathrm{HCMO}+1-$ разрыхляющая $\pi$-орбиталь нафталинового остова.

Важной характеристикой конформеров является значение дипольного момента. Так, „шахматные“ конформеры обладают наибольшим дипольным моментом (около 7 D), в то время как ,заслоненные“ и „плоские“ конформеры имеют меньшие значения дипольных моментов (около 5D). Данный факт может в существенной мере определять различие во взаимодействии конформеров с растворителем, а следовательно, отражаться на распределении конформеров в различных по природе растворителях.

Сравнение строения конформеров в газовой фазе и в водном растворителе показало, что геометрические параметры изменяются незначительно, основные изменения происходят в заместителе $-\mathrm{SO}_{2} \mathrm{NH}_{2}$. Также произошло заметное изменение дипольных моментов конформеров, которое связано с перераспределением 


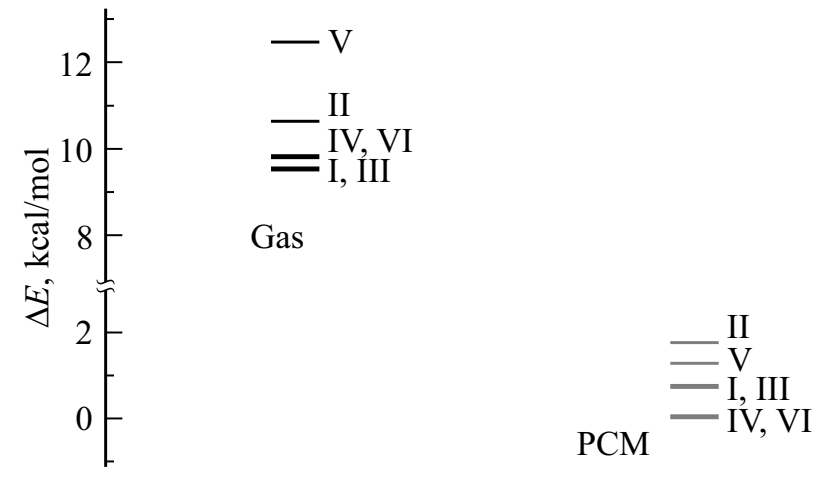

Рис. 3. Энергии конформеров молекулы дансиламида в газовой фазе (Gas) и водном растворителе (PCM), рассчитанные методом DFT(B3LYP)/cc-pVTZ.

электронной плотности в молекуле под влиянием растворителя (рис. 1).

На рис. 3 показаны относительные энергии конформеров в газовой фазе и в водном растворителе. Видно, что энергии всех конформеров в водной среде существенно понижаются по сравнению с газовой фазой (около $9 \mathrm{kcal} / \mathrm{mol}$ ). Кроме того, в растворителе происходит изменение относительной энергии конформеров и конформационного состава. „Шахматные“ конформеры IV и VI, обладающие наибольшим дипольным моментом, стабилизируются в среде полярного растворителя, и их энергия становится ниже энергий ,заслоненных“ конформеров I и III. По этой же причине происходит изменение относительных энергий „плоских“ конформеров II и V. Конформер II с наименьшим дипольным моментом в водном растворителе имеет самую высокую относительную энергию. Как видно из рис. 3, различие в энергии шести конформеров дансиламида в растворителе не превышает $2 \mathrm{kcal} / \mathrm{mol}$, что указывает на возможность их сосуществования в водной среде.

\section{Электронные спектры поглощения конформеров молекулы дансиламида}

Поскольку в эксперименте получены ЭСП сильно разбавленных водных растворов дансиламида, которые, вероятно, содержат конформационную смесь, то для их интерпретации нами были рассчитаны и сопоставлены друг с другом спектры шести конформеров дансиламида в газовой фазе и водной среде в рамках метода TDDFT(B3LYP)/6-311++G**. На рис. 4 в виде диаграмм представлены теоретические ЭСП. Из рисунка видно, что $\lambda_{1}$ в ЭСП конформеров дансиламида варьирует от 355 до $363 \mathrm{~nm}$ в газовой фазе и от 383 до $399 \mathrm{~nm}$ в водном растворителе.

Наибольшее значение $\lambda_{1}$ имеют „шахматные“ конформеры I и III, у которых разность энергий граничных орбиталей минимальна $(4.01 \mathrm{eV}$ - газ, $3.87 \mathrm{eV}$ - вода). Наименьшее значение $\lambda_{1}$ имеют „плоские“ конформеры
II и V, у которых разность энергий граничных орбиталей максимальна $(4.08 \mathrm{eV}$ - газ, $3.97 \mathrm{eV}$ - вода).

Из рисунка видно, что положения трех полос в диапазоне 250-420 nm ЭСП в растворителе существенно сдвинуты в длинноволновую область спектра относительно полос в газовой фазе (батохромный эффект). Кроме того, по сравнению с газовой фазой полоса, соответствующая $\lambda_{1}$, в растворителе существенно расширяется за счет различного взаимодействия конформеров дансиламида с растворителем. Для двух других полос этот эффект выражен слабее. Полоса с $\lambda_{1}$ в основном обусловлена переходом ВЗМО $\rightarrow \mathrm{HCMO}$ (рис. 2), полоса с $\lambda_{2}-$ переходом ВЗМО $\rightarrow \mathrm{HCMO}+1$, полоса с $\lambda_{3}-$ переходом В3МО-1 $\rightarrow$ НСМО (таблица).

\section{Экспериментальный спектр поглощения водного раствора дансиламида}

Как отмечалось ранее, в литературе имеются сведения о спектрах поглощения дансиламида, находящегося в различных протонных и апротонных растворителях, однако подобную информацию для водного раствора нам найти не удалось. Вероятно, это связано с крайне низкой растворимостью дансиламида в воде. В нашей работе показано, что подобная растворимость в воде является достаточной для регистрации ЭСП. На рис. 5 представлены зарегистрированные нами спектры поглощения водных растворов дансиламида с концентрациями $4 \cdot 10^{-4} \mathrm{~mol} / \mathrm{l}$ и $2 \cdot 10^{-4} \mathrm{~mol} / \mathrm{l}$. Максимум полосы поглощения $\lambda_{1 \max }$ водного раствора дансиламида составил $326 \mathrm{~nm}$, что согласуется с литературными данными для других протонных растворителей [8]. Также на графике обозначены максимумы полос поглощения $\lambda_{2 \max }$ и $\lambda_{3 \max }$, которые составили 214 и $244 \mathrm{~nm}$.

Качественно теоретические ЭСП в водной среде (рис. 4) соответствуют экспериментальным спектрам.

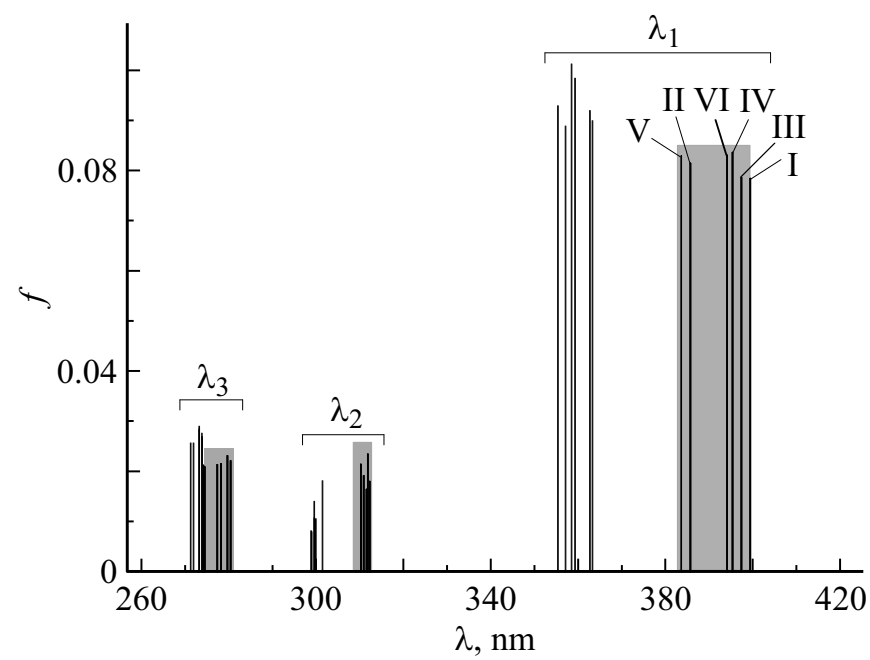

Рис. 4. Вычисленные ЭСП конформеров молекулы дансиламида в газовой фазе и в водном растворителе (линии на сером фоне). 
Результаты расчетов ЭПС конформеров дансиламида $(f-$ сила осциллятора)

\begin{tabular}{|c|c|c|c|c|c|}
\hline \multirow{2}{*}{ Конформер } & \multicolumn{2}{|c|}{ Газовая фаза } & \multicolumn{2}{|c|}{ Водный раствор } & \multirow{2}{*}{$\begin{array}{c}\text { МО, вовлеченные } \\
\text { в электронный переход }\end{array}$} \\
\hline & $\lambda, \mathrm{nm}(\Delta E, \mathrm{eV})$ & $f$ & $\lambda, \mathrm{nm}(\Delta E, \mathrm{eV})$ & $f$ & \\
\hline I & $\begin{array}{l}362.8(3.42) \\
299.1(4.15) \\
274.0(4.53)\end{array}$ & $\begin{array}{l}0.0920 \\
0.0080 \\
0.0277\end{array}$ & $\begin{array}{l}399.3(3.10) \\
311.5(3.98) \\
280.4(4.42)\end{array}$ & $\begin{array}{l}0.0785 \\
0.0165 \\
0.0222\end{array}$ & $\begin{array}{l}\mathrm{HOMO} \rightarrow \text { LUMO } \\
\mathrm{HOMO} \rightarrow \text { LUMO }+1 \\
\mathrm{HOMO}-1 \rightarrow \text { LUMO }\end{array}$ \\
\hline II & $\begin{array}{l}355.5(3.49) \\
299.7(4.14) \\
271.4(4.57)\end{array}$ & $\begin{array}{l}0.0930 \\
0.0141 \\
0.0257\end{array}$ & $\begin{array}{l}383.6(4.00) \\
310.2(4.52) \\
274.2(4.73)\end{array}$ & $\begin{array}{l}0.0830 \\
0.0215 \\
0.0213\end{array}$ & $\begin{array}{l}\mathrm{HOMO} \rightarrow \text { LUMO } \\
\mathrm{HOMO} \rightarrow \text { LUMO }+1 \\
\mathrm{HOMO}-1 \rightarrow \text { LUMO }\end{array}$ \\
\hline III & $\begin{array}{l}363.4(3,41) \\
298.9(4.15) \\
274.0(4.53)\end{array}$ & $\begin{array}{l}0.0900 \\
0.0270 \\
0.0082\end{array}$ & $\begin{array}{l}397.3(3.12) \\
310.2(4.00) \\
278.2(4.69)\end{array}$ & $\begin{array}{l}0.0787 \\
0.0174 \\
0.0216\end{array}$ & $\begin{array}{l}\mathrm{HOMO} \rightarrow \text { LUMO } \\
\mathrm{HOMO} \rightarrow \text { LUMO }+1 \\
\mathrm{HOMO}-1 \rightarrow \text { LUMO }\end{array}$ \\
\hline IV & $\begin{array}{l}358.6(3.46) \\
300.0(4.13) \\
273.2(4.54)\end{array}$ & $\begin{array}{l}0.1013 \\
0.0106 \\
0.0291\end{array}$ & $\begin{array}{l}395.3(3.14) \\
312.2(3.97) \\
279.7(4.43)\end{array}$ & $\begin{array}{l}0.0837 \\
0.0181 \\
0.0232\end{array}$ & $\begin{array}{l}\mathrm{HOMO} \rightarrow \text { LUMO } \\
\mathrm{HOMO} \rightarrow \text { LUMO }+1 \\
\mathrm{HOMO}-1 \rightarrow \text { LUMO }\end{array}$ \\
\hline V & $\begin{array}{l}357.2(3.47) \\
301.6(4.11) \\
272.1(4.56)\end{array}$ & $\begin{array}{l}0.0889 \\
0.0182 \\
0.0257\end{array}$ & $\begin{array}{l}385.7(3.21) \\
311.8(3.98) \\
274.5(4.52)\end{array}$ & $\begin{array}{l}0.0815 \\
0.0235 \\
0.0210\end{array}$ & $\begin{array}{l}\mathrm{HOMO} \rightarrow \text { LUMO } \\
\mathrm{HOMO} \rightarrow \text { LUMO }+1 \\
\mathrm{HOMO}-1 \rightarrow \text { LUMO }\end{array}$ \\
\hline VI & $\begin{array}{l}359.4(4.56) \\
299.7(4.14) \\
273.3(4.54)\end{array}$ & $\begin{array}{l}0.0985 \\
0.0108 \\
0.0285\end{array}$ & $\begin{array}{l}394.0(3.15) \\
310.9(3.99) \\
277.3(4.47)\end{array}$ & $\begin{array}{l}0.0831 \\
0.0192 \\
0.0214\end{array}$ & $\begin{array}{l}\mathrm{HOMO} \rightarrow \text { LUMO } \\
\mathrm{HOMO} \rightarrow \text { LUMO }+1 \\
\mathrm{HOMO}-1 \rightarrow \text { LUMO }\end{array}$ \\
\hline
\end{tabular}

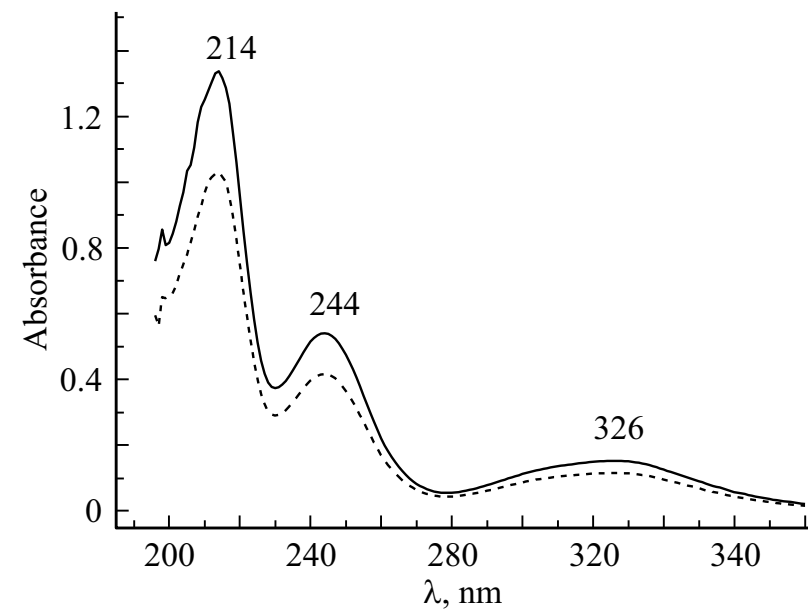

Рис. 5. Спектры поглощения водных растворов дансиламида: $0.0004 \mathrm{~mol} / 1$ (сплошная кривая), $0.0002 \mathrm{~mol} / 1$ (штриховая кривая).

Так, расстояние между полосами при $\lambda_{1}$ и $\lambda_{2}$ больше, чем между полосами при $\lambda_{2}$ и $\lambda_{3}$. Это коррелирует с величинами $\Delta E-$ разностями энергий $\mathrm{MO}$ (рис. 2), вовлеченных в соответствующие электронные переходы $\left(\Delta E_{\text {Взмо-нсмо }}=3.7 \mathrm{eV}, \Delta E_{\text {Взмо-нСмО }+1}=4.7 \mathrm{eV}\right.$, $\left.\Delta E_{\text {ВзМО-1-НСМО }}=5.0 \mathrm{eV}\right)$.

Полосы экспериментального ЭСП с $\lambda_{2 \max }$ и $\lambda_{3 \max }$ более узкие, чем полоса $\lambda_{1 \text { max }}$, что соответствует представлению о наличии конформеров дансиламида в водном растворе.

\section{Зависимость рассчитанных ЭСП от выбранного функционала электронной плотности}

Сравнение экспериментального ЭСП водного раствора дансиламида и его теоретических аналогов, полученных с использованием метода TDDFT с функционалом B3LYP, показало значительное отличие в абсолютных значениях длин волн поглощения. Так, разность значений $\lambda_{1 \max }$ в экспериментальном ЭСП для водного

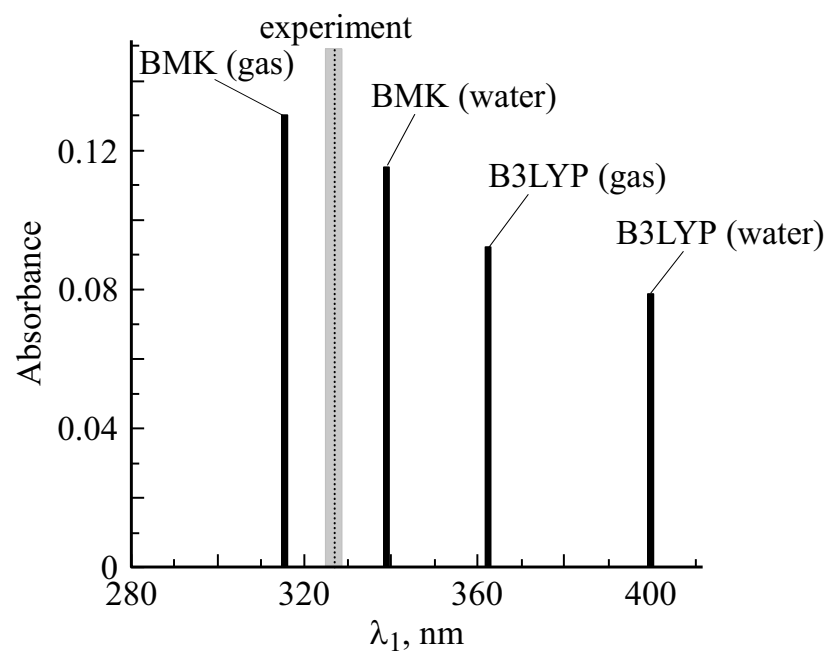

Рис. 6. Экспериментальное значение $\lambda_{\max }$ для водного раствора дансиламида (experiment) и рассчитанные значения $\lambda_{\max }$ для свободного конформера I (gas) и конформера I с учетом водного растворителя (water). 
раствора дансиламида (рис. 5) и $\lambda_{1}$ в рассчитанном ЭСП для газовой фазы (рис. 4) составляет около $30 \mathrm{~nm}$. При учете влияния водного растворителя в рамках модели РСМ эта разность возрастает до $60 \mathrm{~nm}$ (рис. 6).

Нами была проверена предсказательная способность различных функционалов метода DFT для описания ЭСП. Было выбрано несколько наиболее используемых функционалов DFT, а именно B3LYP, B97D, M06, BMK, PBE1PBE. Все расчеты проводились методом TDDFT с базисом 6-311++ G $^{* *}$. В результате были получены следующие значения $\lambda_{1}$ для конформера I свободной молекулы дансиламида: B3LYP - $363 \mathrm{~nm}$, B97D - $434 \mathrm{~nm}$, M06 - $346 \mathrm{~nm}$, BMK - $316 \mathrm{~nm} \mathrm{и}$ PBE1PBE - $350 \mathrm{~nm}$.

Таким образом, рассчитанные величины $\lambda_{1}$, так же как и энергии граничных орбиталей, зависят от вида функционала электронной плотности. На рис. 6 показана диаграмма, в которую включены значения $\lambda_{1}$, рассчитанные с использованием функционалов BMK и B3LYP для свободного конформера I и этого конформера в водной среде. Видно, что функционал ВМК практически воспроизводит экспериментальное значение максимума полосы $\lambda_{1 \max }$ водного раствора дансиламида.

\section{Заключение}

Рассмотрены характеристики шести конформеров молекулы дансиламида: геометрическое строение, энергии и вид МО, вовлеченных в электронные переходы, дипольные моменты, относительная энергия конформеров как в свободном состоянии, так и в водной среде (РСМ).

Энергии всех конформеров в водной среде существенно понижаются по сравнению с газовой фазой (около $9 \mathrm{kkal} / \mathrm{M})$. В растворителе происходит изменение относительной энергии конформеров и конформационного состава. Конформеры, обладающие наибольшим дипольным моментом, стабилизируются в среде полярного растворителя.

Зарегистрированы ЭСП водных растворов дансиламида с концентрациями $4 \cdot 10^{-4}$ и $2 \cdot 10^{-4} \mathrm{M} / 1$.

Рассчитаны ЭСП шести конформеров дансиламида в газовой фазе и водной среде в рамках метода TDDFT(B3LYP)/6-311++G**. Положения полос ЭСП в растворителе существенно сдвинуты в длинноволновую область спектра относительно полос в газовой фазе. Расстояние между полосами ЭСП коррелирует с величинами $\Delta E-$ разностью энергий $\mathrm{MO}$, вовлеченных в соответствующие электронные переходы. Показано согласие на качественном уровне теоретических ЭСП в водной среде и экспериментальных спектров растворов дансиламида.

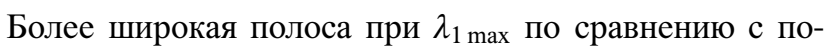
лосами при $\lambda_{2 \max }$ и $\lambda_{3 \max }$ соответствует представлению о наличии конформеров дансиламида в водном растворе.

Рассмотрено влияние различных функционалов (B3LYP, B97D, M06, BMK и PBE1PBE) метода DFT на положение полосы $\lambda_{1}$ в рассчитанных ЭСП. Показано, что функционал ВМК воспроизводит значение максимальной длины волны поглощения для водного раствора дансиламида наиболее точно.

Авторы выражают благодарность проф. Кустовой Т.П. за помощь в проведении экспериментальной части работы.

Работа выполнена при финансовой поддержке РФФИ (проект № 16-33-00386 мол_а).

\section{Список литературы}

[1] Shinoda S., Okazaki T., Player T.N., Misaki H., Hori K., Tsukube H. // J. Org. Chem. 2005. V. 70. N 5. P. 1835. doi $10.1021 /$ jo0478297

[2] Nair S.K., Elbaum D., Christianson D.W. // J. Biol. Chem. 1996. V. 271. N 2. P. 1003. doi 10.1074/jbc.271.2.1003

[3] Matsuoka K., Arai H., Oka H., Koyama T., Hatano K. // ACS Macro Lett. 2012. V. 1. N 2. P. 266. doi $10.1021 / \mathrm{mz} 200135 \mathrm{y}$

[4] ChEMBL (chemical database of bioactive molecules) [Электронный ресурс] Режим доступа: https://www.ebi.ac.uk/chembl/

[5] Supuran C.T., Scozzafava A., Briganti F., Clare B.W. // J. Med. Chem. 2000. V. 43. N 9. P. 1793. doi $10.1021 / \mathrm{jm} 9903693$.

[6] National Center for Biotechnology Information. PubChem BioAssay Database; AID=686978 [Электронный ресурс] Режим доступа: https://pubchem.ncbi.nlm.nih.gov/bioassay/686978.

[7] Gröneberg S., Stubbs M.T., Klebe G. // J. Med. Chem. 2002. V. 45. N 17. P. 3588. doi 10.1021/jm011112j

[8] Tewari N., Joshi N.K., Rautela R., Gahlaut R., Joshi H.C., Pant S. // J. Mol. Liq. 2011. V. 160. P. 150. doi 10.1016/j.molliq.2011.03.008

[9] Барановский В.И. // Опт. и спектр. 2007. Т. 103. № 4. C. 560. Baranovski V.I. // Opt. Spectrosc. 2007. V. 103. N 4. P. 540. doi 10.1134/S0030400X07100050

[10] Гиричева Н.И., Лапыкина Е.А., Федоров М.С., Петрова Д.А. // Журн. структ. химии. 2015. Т. 56. № 4. С. 669. Giricheva N.I., Lapykina E.A., Fedorov M.S., Petrova D.A. // J. Struct. Chem. 2015. V. 56. N 4. P. 619. doi $10.1134 / \mathrm{S} 0022476615040034$

[11] Frisch M.J., Trucks G.W., Schlegel H.B., Scuseria G.E., Robb M.A., Cheeseman J.R., Scalmani G., Barone V., Mennucci B., Petersson G.A., Nakatsuji H., Caricato M., Li X., Hratchian H.P., Izmaylov A.F., Bloino J., Zheng G., Sonnenberg J.L., Hada M., Ehara M., Toyota K., Fukuda R., Hasegawa J., Ishida M., Nakajima T., Honda Y., Kitao O., Nakai H., Vreven T., Montgomery J.A., Peralta J.E., Ogliaro F., Bearpark M., Heyd J.J., Brothers E., Kudin K.N., Staroverov V.N., Kobayashi R., Normand J., Raghavachari K., Rendell A., Burant J.C., Iyengar S.S., Tomasi J., Cossi M., Rega N., Millam J.M., Klene M., Knox J.E., Cross J.B., Bakken V., Adamo C., Jaramillo J., Gomperts R., Stratmann R.E., Yazyev O., Austin A.J., Cammi R., Pomelli C., Ochterski J.W., Martin R.L., Morokuma K., Zakrzewski V.G., Voth G.A., Salvador P., Dannenberg J.J., Dapprich S., Daniels A.D., Farkas Ö., Foresman J.B., Ortiz J.V., Cioslowski J., Fox D.J. Gaussian Inc., Wallingford CT. 2009. 
[12] Kulinich A.V., Mikitenko E.K., Ishchenko A.A. // Opt. Spectrosc. 2015. V. 119. N 1. P. 39. doi 10.1134/S0030400X15070164

[13] Gökce H., Bahçeli S. // Opt. Spectrosc. 2014. V. 117. N 1. P. 82. doi 10.1134/S0030400X14040110

[14] Aksakal N.E., Bayar M., Dumrul H., Atilla D., Chumakov Yu., Yuksel F. Polycyclic Aromatic Compounds. 2017. doi 10.1080/10406638.2017.1327871

[15] Basheer S.M., Bhuvanesh N.S.P., Sreekanth A. // J. Fluorine Chemistry. 2016. V. 191. P. 129. doi 10.1016/j.jfluchem.2016.10.005

[16] Karabacak M., Cinar M., Kurt M., Poiyamozhi A., Sundaraganesan N. // Spectrochim. Acta. A. 2014. V. 117. P. 234. doi 10.1016/j.saa.2013.07.095

[17] Федоров М.С., Гиричева Н.И., Лапыкина Е.А., Суворова О.A. // Опт. и спектр. 2017. Т. 123. № 2. C. 217. Fedorov M.S., Giricheva N.I., Lapykina E.A., Suvorova O.A. // Opt. Spectrosc. 2017. V. 123. N 2. P. 231. doi 10.1134/S0030400X17080045

[18] Zhurko G.A., Zhurko D.A. Chemcraft Program. [Электронный ресурс] Режим доступа: http://www.chemcraftprog.com. 